\title{
MRI protocol technique in the optimal therapeutic strategy of non-functioning pituitary adenomas
}

Gustavo Soto-Ares, Christine Cortet-Rudelli ${ }^{1}$, Richard Assaker ${ }^{2}$, Arnaud Boulinguez, C Dubest ${ }^{1}$, Didier Dewailly ${ }^{1}$ and Jean Pierre Pruvo

Department of Neuroradiology, Hôpital Roger Salengro, ${ }^{1}$ Department of Endocrinology, Clinique Marc Linquette and ${ }^{2}$ Department of Neurosurgery, Hôpital Roger Salengro, Chru Lille, France

(Correspondence should be addressed to Gustavo Soto-Ares, Department of Neuroradiology, Hôpital Roger Salengro, Chru Lille 59037, Lille, France; Email: gsotoares@chru-lille.fr)

\begin{abstract}
Objectives and Design: We performed a prospective study using magnetic resonance imaging (MRI) at regular post-operative intervals in non-irradiated patients with non-functioning pituitary adenomas (NFAs) to assess the frequency of tumoral regrowth and recurrences, in order to define the indications of post-operative radiotherapy.

Patients and Methods: Fifty-one patients aged 25-80 years (mean, 55.6 \pm 12.3 years) were included. Post-operative MRIs were performed 3-12 months (mean, 5.2 \pm 1.7 months) after surgery, 6 months later and then, every 12-18 months for at least 2 years. The mean post-operative

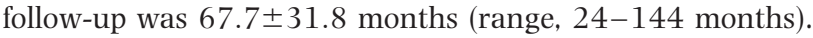

Results: In 17 patients (33\%, group I) no tumoral residue was observed on post-operative MRIs and no tumoral recurrence was diagnosed. Tumour regrowth was detected in 13 of the 34 patients (38.2\%) with post-operative tumoral residue (group II), 7-66 months (mean, 27.3 \pm 17.3 months) after surgery. In this group, Kaplan-Meier analysis showed $78.8 \%$ recurrence free survival at 2 years and $60.9 \%$ at 5 years. Patients with tumoral regrowth had higher mean residual tumoral volume than patients without any tumoral regrowth in the group II $\left(258 \pm 165 \mathrm{vs} 163 \pm 165 \mathrm{~mm}^{3}, P=0.05\right)$. Conclusions: We suggest a MRI protocol that includes, a 4- to 6-, 12- and 24-month post-operative MRI for every patient. When no tumoral residue is seen, pituitary radiotherapy is useless. MRI must be repeated 3, 5 and 10 years after surgery to eliminate late recurrence. The observed frequency of tumoral regrowth in patients with tumoral residue does not justify systematic post-operative radiotherapy. It should be performed only when tumoral regrowth is proved by a yearly MRI survey.
\end{abstract}

European Journal of Endocrinology 146 179-186

\section{Introduction}

Trans-sphenoidal surgery is the primary treatment for clinically non-functioning pituitary adenomas (NFAs). However, these tumours are frequently invasive and tumoral post-operative residues are frequent. Tumour regrowth/recurrence rates following surgery have been reported to be $18 \%$ at 5 years and $41-44 \%$ at 10 years $(1,2)$ when no post-operative radiotherapy is performed. However, in these studies, diagnosis was mainly made using clinical symptoms of mass effect or CT scans. MRI was not systematically performed and there was no prospective protocol for follow-up imaging. This may alter the sensitivity of detection of recurrences, underestimate their true frequency and explain some late diagnoses ( $>10$ years).

In NFAs, pituitary radiotherapy significantly reduces the risk of tumour regrowth with recurrence-free survival of between 87.5 and $97 \%$ at 10 years and between 72 and $92 \%$ at 20 years (3-9). However, irradiation results in hypopituitarism in at least $40-50 \%$ of the patients $(4,10,11)$ and visual complications are reported in $1-3 \%(4,10,12)$. In spite of a low incidence, the relative risk of patients developing severe secondary tumours, such as gliomas, astrocytoma or sarcoma, is significantly increased $(12-14)$. So, the indication of post-operative radiotherapy remains controversial. Some authors advocate systematic post-operative radiotherapy for patients with NFAs (2). Others recommend it only for patients with post-operative residual tumour $(4,5,9)$. Bradley et al. (15) studied selected patients who had not undergone early irradiation. They identified a subgroup of patients with a good prognosis (complete surgical removal of the tumour, absence of macroscopic or histological invasion of para-sellar structures). In 
these patients, post-operative irradiation is indicated only when recurrence is proven.

To be able to determine the risk-benefit ratio of postoperative radiotherapy it is essential to establish the true frequency of post-operative tumoral regrowths and recurrences. We therefore performed a prospective study using MRI at regular follow-up intervals. Patients were scanned before and 3-7 months after surgery. This exam was then systematically repeated 6 months later and every 12-18 months thereafter. None of the patients observed in this study underwent systematic pituitary radiotherapy. We tried to identify factors that could predict recurrence or tumour regrowth in order to select patients who should receive radiotherapy.

\section{Patients and methods}

Fifty-one patients (22 males and 29 females) with clinically non-functioning pituitary adenoma, aged 25-80

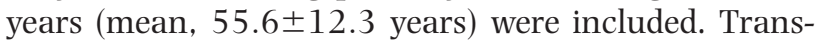
sphenoidal surgery was performed in 48 patients. One patient had trans-frontal surgery. The two surgical approachs were performed in two patients. None of them had systematic post-operative pituitary radiotherapy. Forty-one percent of the patients had headaches. Loss of visual acuity and visual field defects were found in 56.9 and $90.2 \%$ of them, respectively. In $17.6 \%$ of the patients, the adenoma was diagnosed accidentally whilst undergoing MRI or CT scans for other indications. High plasma levels of a-subunit, follicle stimulating hormone (FSH) and/or luteinizing hormone (LH) levels were found in $13.7 \%$ of the patients. The immunostaining characteristics of the 48 evaluable adenomatous tissues were: gonadotrop $(n=29)$, non-functioning $(n=14)$ and silent corticotrop adenomas $(n=5)$. Gonadotrop, corticotrop and thyreotrop deficiency were diagnosed in 47.1, 43.1 and $27.5 \%$ of the patients, respectively.

Patients were scanned pre-operatively. The first postoperative MRI was performed at a mean of $5.2 \pm 1.7$ months after surgery (at $3-4$ months: $n=18$; at 5-6 months: $n=27$; at 7 months: $n=5$; at 12 months: $n=1$ ). The second scan was performed 6 months later and then repeated every 12-18 months
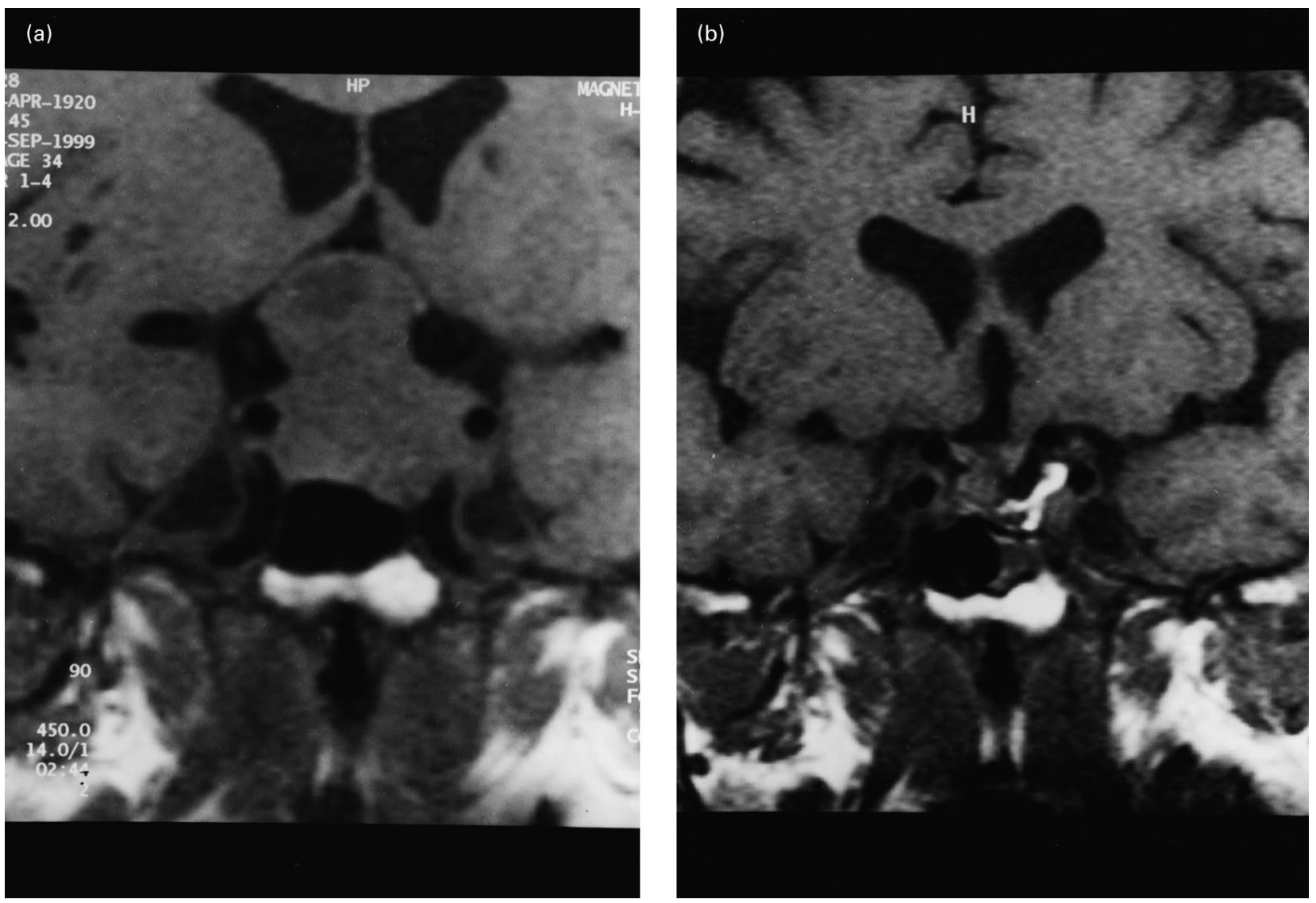

Figure $1 \mathrm{MRI}$ findings in residual tumour. Coronal T1 (450/14/2) before surgery (a) shows an isointense mass with suprasellar extension and optic chiasma compression. In post-operative control (b), the residual tumour has the same signal characteristics. Note the hyperintensity of the surgical material at the left side of the sella. 
for at least 2 years. The mean post-operative follow up was $67.7 \pm 31.8$ months (range, $24-144$ months). MRI has been available in our institution since 1990. Until 1994, the subjects were scanned with a $0.5 \mathrm{~T}$ Magnetom Vision imager using a standard head coil. After 1994, MRI examinations were performed in a $1 \mathrm{~T}$ Magnetom Expert imager (Siemens, Erlangen, Germany). Imaging studies included sagittal T1-weighted (600/14/1: repetition time/echo time/ excitations) images and coronal T1- and TSE T2-weighted (4000/120/2) MR images. Coronal FLASH 2D dynamic series after contrast (180/4/1), were followed by sagittal and coronal post-contrast T1-weighted images. Every MRI was reviewed by two neuroradiologists, one neurosurgeon and two endocrinologists to assess invasion of sphenoidal, cavernous sinus and/or opto chiasmatic citern in pre-operative scans, and to detect residual tumour and tumour regrowth in serial post-operative images. Invasion of cavernous sinus was diagnosed according to the classification proposed by Cottier et al. (16). Residual tumour was defined as a nodular mass with signal characteristics identical to those of the pre-operative adenomatous tissue. All cases with uncertain MRI diagnosis of residual tumour were included in the group of patients with residual tumour. Tumour regrowth was defined as an increase of the tumoral residue volume. Recurrence was defined by the appearance of adenomatous tissue with the same signal as the pre-operative mass in patients without any tumoral residue on the first post-operative MRI(s).

The choice of complementary treatment in cases of recurrences was made in multidisciplinary decision on a case-by-case basis.

Recurrence-free survival was measured by the actuarial method of Kaplan \& Meier (17).

\section{Results}

\section{MRI pre-operative features}

In $82 \%$ of cases, the tumour was hypointense in T1-weighted images and hyperintense in T2. In the other cases, the tumours were isointense compared with the normal pituitary gland. The images of all the tumours were enhanced after contrast administration.
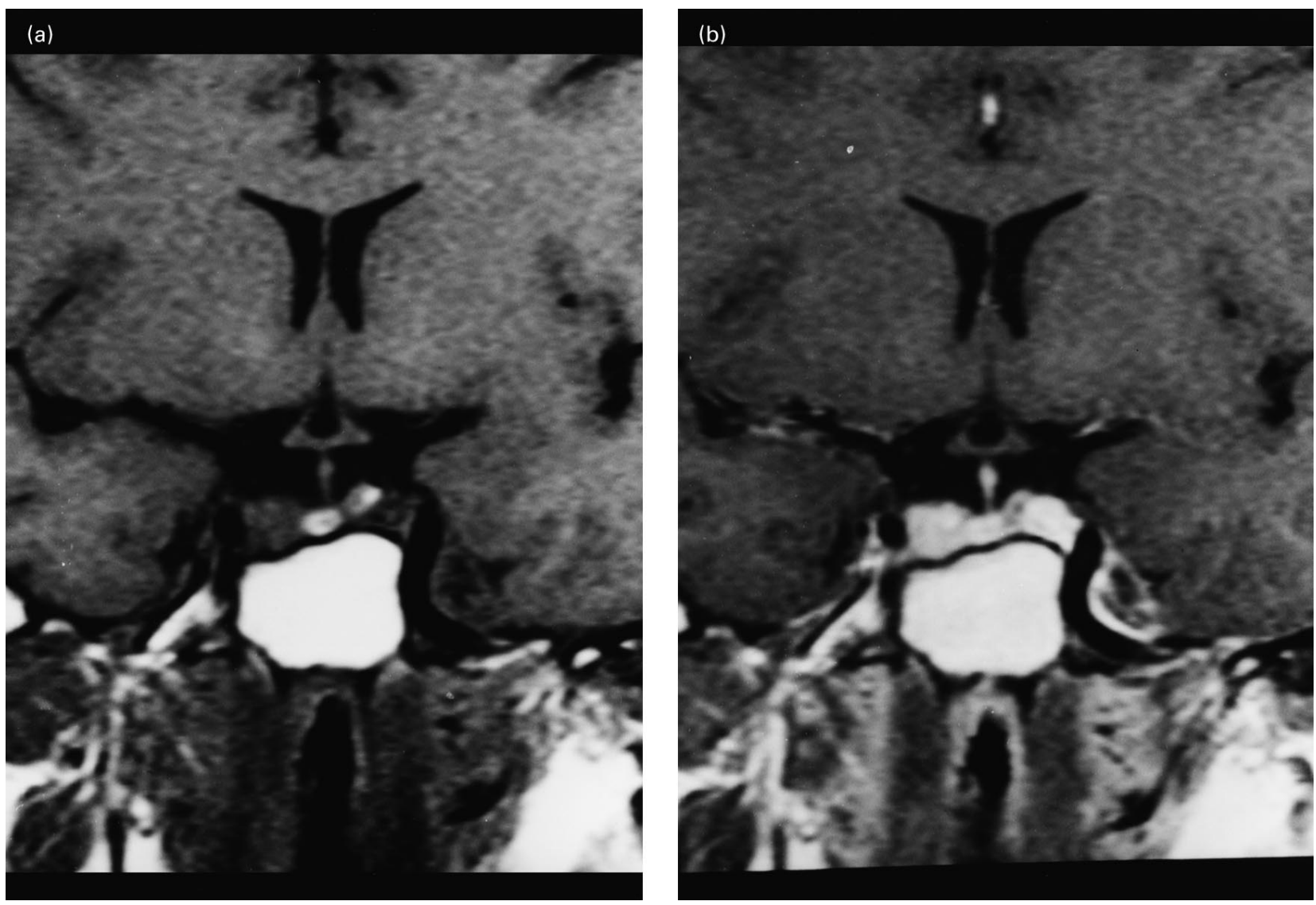

Figure 2 Residual tumour enhancement. Coronal T1-weighted image before (a) and after (b) contrast. In all cases, the image of the residual tumour is enhanced after contrast administration. The tumour is less enhanced than the pituitary stalk and the neurohypophysis. 

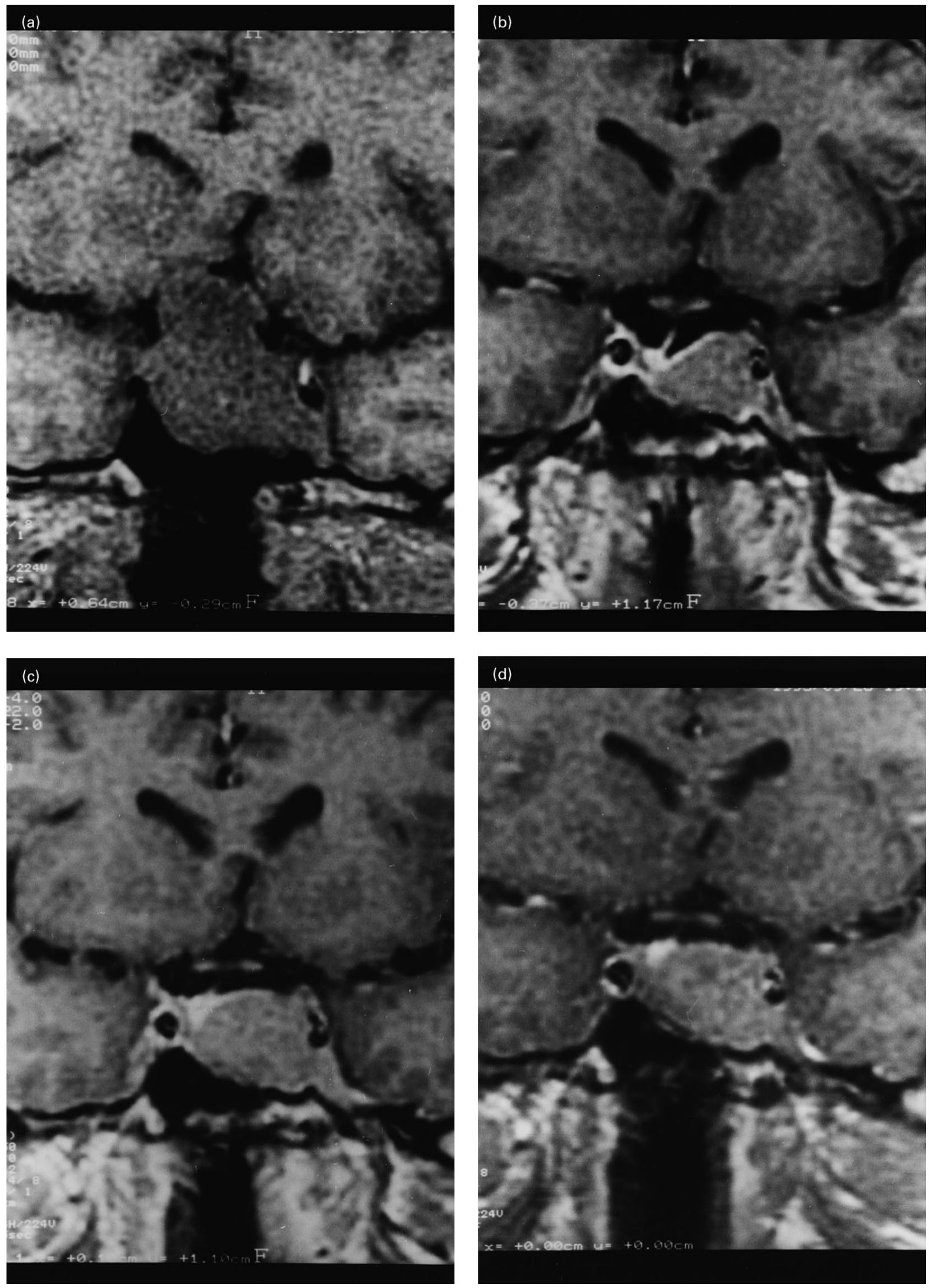

Figure 3 Tumoral recurrence and regrowth. Coronal T1-weighted image before (a) and after (b) surgery. The volume of the residual tumour is important and recurrence at 6 (c) and 12 months (d) is progressive. 

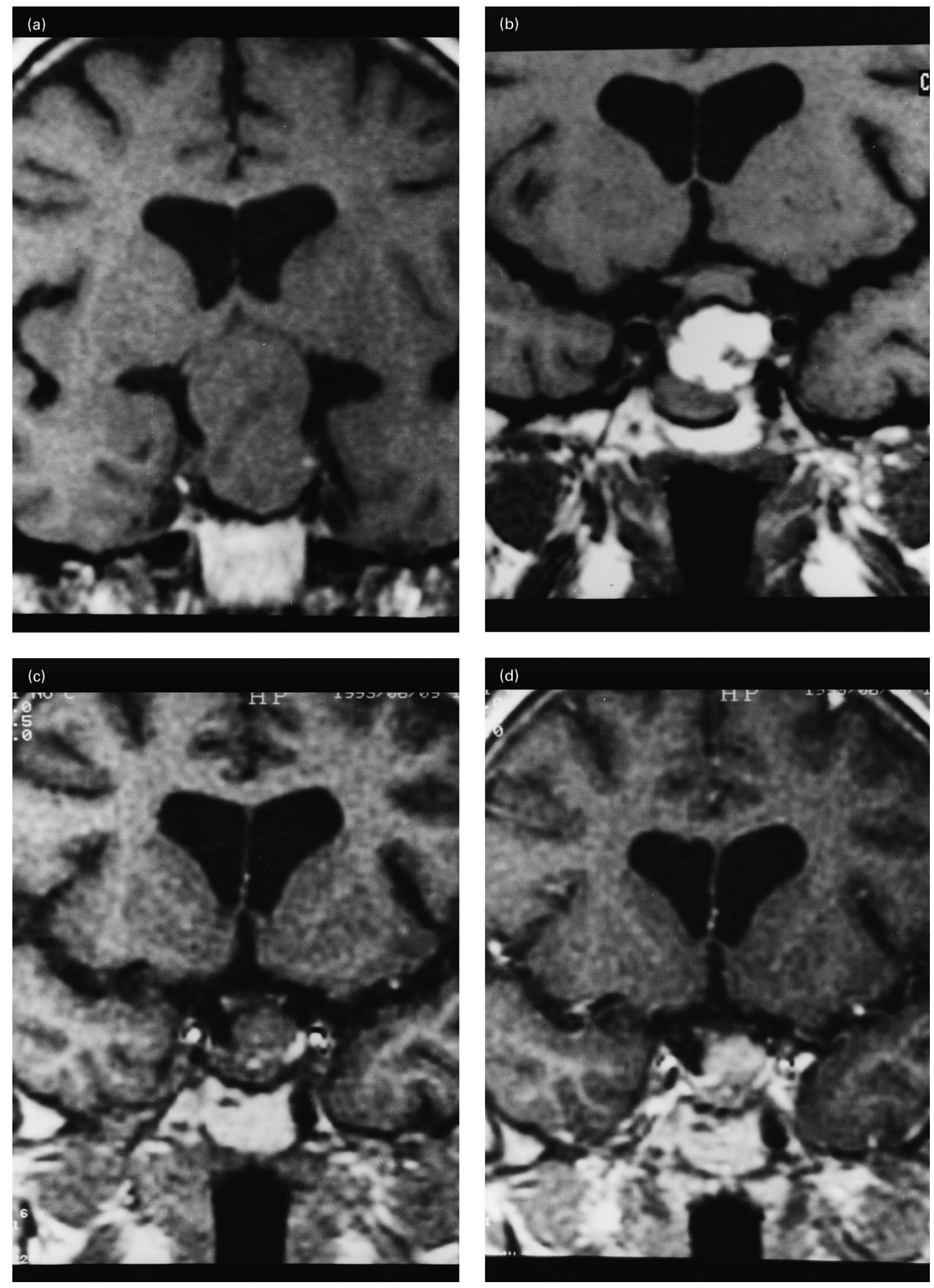

Figure 4 For legend, see next page. 


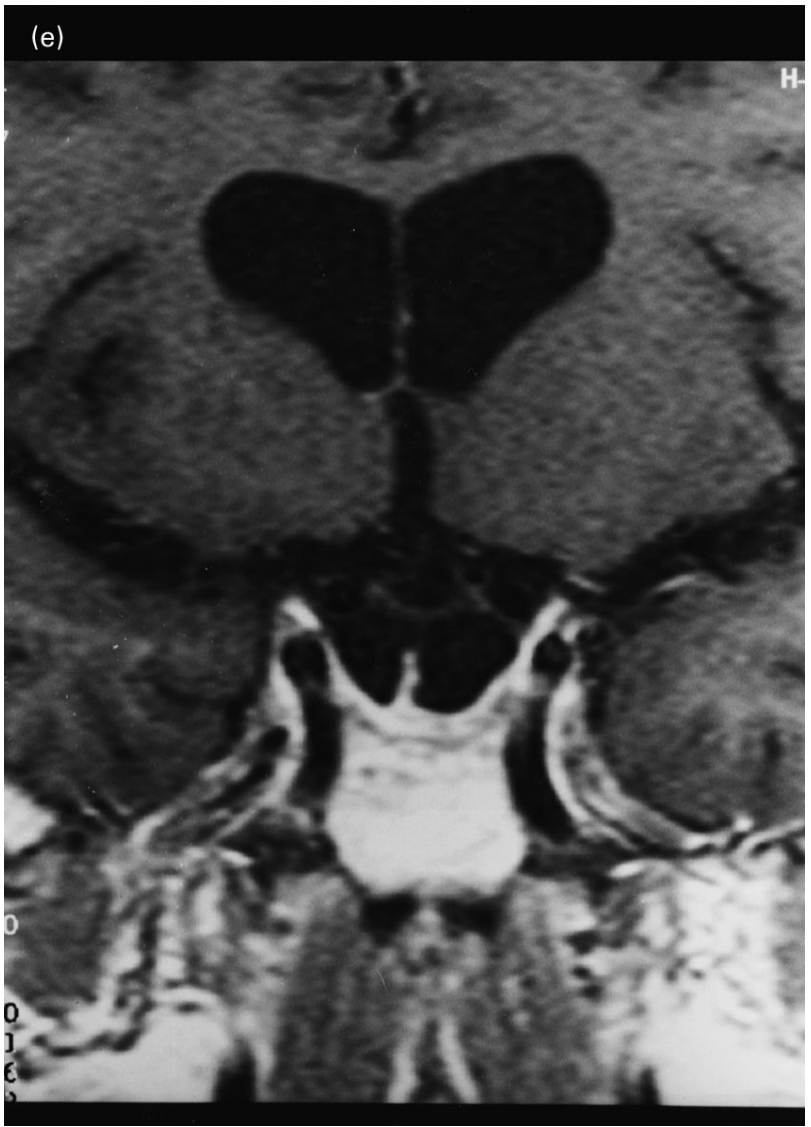

Figure 4 Residual mass operated on a second time. Coronal image before (a) and after surgery (b). Note the post-operative mass effect with the isointense residual tumour within the suprasellar cistern and the hyperintense surgical material. After 24 months, the surgical material is totally resorbed and the residual mass, well limited before (c) and after (d) contrast could be surgically removed. The final control (e) shows the absence of residual tumour.

Invasion of para-sellar structures included the sphenoidal sinus in $14 \%$ of the patients and the cavernous sinus in $45 \%$ patients. Eighty-eight percent of the patients had supra-sellar expansion.

\section{MRI and residual tumour}

Thirty-four patients $(67 \%)$ had a tumoral residue on post-operative MRI(s) (Fig. 1a and b). In 93.5\% of patients, the residual tumour was hypointense in T1-weighted images, after contrast administration the image was enhanced but less so than for the normal pituitary gland in all cases (Fig. 2a and b). Forty-five percent of the residual lesions had a heterogeneous signal in $\mathrm{T} 2$.

\section{MRI and tumour recurrence}

The mean follow-up was $67.7 \pm 31.8$ months (range, 24-144 months). Pituitary tumour regrowth occurred in $13 / 51$ of patients $(25.5 \%)$. The tumoral regrowth was detected at a mean of $27.3 \pm 17.3$ months (range, 7-66 months) after surgery. Kaplan-Meier analysis showed $86 \%$ tumoral regrowth-free survival at 2 years (95\% confidence limits, $77-96 \%)$ and $74 \%$ at 5 years $(95 \%$ confidence limits, $56-84 \%)$. The tumoral regrowth was asymptomatic in $9 / 13$ patients $(69 \%)$.

In 17 patients (33\%, group I) no tumoral residue was found in post-operative MRIs. No tumoral recurrence was observed in this group. The frequency of tumoral regrowth was $38.2 \%$ in the group of patients with a post-operative tumoral residue $(n=34$, group II). Kaplan-Meier analysis showed $78.8 \%$ recurrence-free survival at 2 years and $60.9 \%$ at 5 years in patients of the group II. The mean follow up was the same in the two groups ( $66.3 \pm 33.9$ vs $69.8 \pm 30.6$ months).

The neurosurgeon thought that surgical resection was complete for 25 patients but ten of them (40\%) had a residual tumour on the post-operative MRIs. Two of these ten patients had subsequent tumour regrowth. Three patients with suspected incomplete resection had normal post-operative MRIs.

There was no significant relationship between tumoral regrowth and sex, age, immunostaining characteristics of the adenoma, the initial tumoral volume or the MRI signal of the tumour. Patients with tumoral regrowth had higher mean residual tumoral volume than patients without any tumoral regrowth in the group II $(258 \pm 165$ vs $163 \pm$ $165 \mathrm{~mm}^{3}, P=0.05$ ) (Fig. 3a-d).

\section{Complementary treatment}

In cases with tumoral regrowth, 5/13 patients were operated on a second time (Fig. 4a-e); 4/13 patients were operated on and underwent subsequent radiotherapy; 3/13 patients underwent radiotherapy alone. Only one patient was medically treated because of poor clinical status and outcome.

\section{Discussion}

To our knowledge, this is the first prospective study evaluating the frequency of tumoral regrowth and recurrence in non-irradiated patients with NFAs, using MRI at regular post-operative follow-up intervals. We showed that tumoral regrowth was detected only among patients with a tumoral residue. The lesion was hypointense $(93.5 \%)$ or isointense $(6.5 \%)$ on T1-weighted images, with low enhancement after contrast administration when compared with normal pituitary gland on post-operative MRIs. A meticulous MRI technique is necessary to obtain a good delineation of the residual tumour and to demonstrate the tumoral regrowth. It consists of thin T1- and T2-weighted images obtained in coronal and sagittal planes $(3 \mathrm{~mm}$ thick) with a high resolution technique, before and 
after contrast administration. The post-operative mass effect can obscure remaining tumoral tissue and may alter the sensitivity of detection of residual tumour. Since stabilisation of the post-surgical changes occur by the 4 th month $(18,19)$, the optimal time to perform the first post-operative MRI should be 4-6 months after surgery. If the diagnosis of residual tumour is uncertain, MRI has to be repeated 6 and sometimes 12 months later, before concluding.

We demonstrated that surgeon assessment of complete tumoral removal was poor compared with MRI post-operative findings. Forty percent $(n=10)$ of the patients deemed to have macroscopic per-operative complete surgical removal $(n=25)$ were shown to have tumoral residue on post-operative MRIs. Two of them $(20 \%)$ had subsequent tumoral regrowth. In this setting, our results clarify the statement of Turner et al. (2) who demonstrated that the surgeon's assessment of complete surgical removal was unrelated to recurrence.

Tumoral regrowth-free survival at 5 years in our study seems to be comparable with results obtained by Turner et al. (2). However the mean delay of tumoral regrowth was $27.7 \pm 17.3$ months in our series and 5.4 years in their study (2). Some of their patients had CT scans only, which is less reliable than MRI at detecting tumoral residue and/or recurrence. Moreover, there was no regular imaging follow-up. This could explain the longer delay in diagnosing recurrence observed by these authors. If our results are confirmed with a longer follow-up and a larger number of patients, it would suggest that yearly MRI allows earlier, more reliable diagnosis of tumoral regrowth. Tumoral regrowth has been reported to occur several years ofter surgery among non-irradiated patients with a post-operative adenomatous residue (1, 3, 4). So, yearly clinical and morphological follow-up has to be performed for life in these patients.

According to previous data, we found no significant relationship between tumoral regrowth and sex, age, immunostaining characteristics of the adenoma or initial tumoral volume. The volume of the residual tumour was the only morphological factor indicating more frequent recurrences.

We think that the frequency of recurrences observed in our study does not justify systematic immediate post-operative radiotherapy for all patients with postoperative adenomatous residue. Because of its potential complications, it should be performed only when tumoral regrowth is proven. However, if the adenomatous residue is voluminous and close to the optic nerves/chiasma, the indication of systematic postoperative radiotherapy has to be discussed. Indeed, in the event of a tumoral regrowth the risk of visual worsening may be more important than the potential side effects of pituitary irradiation.

In our study, no true recurrence was observed. No recurrence was diagnosed in patients in whom MRI performed at 6, 12 and 24 months after surgery failed to detect a tumoral residue and so, radiotherapy would be pointless in these patients. Because MRI has only been available since 1990 in our department, the length of follow-up in our study is not sufficient to definitively conclude. In the absence of clinical or biological signs of recurrence, we suggest that MRI should be repeated at intervals of 3,5 and 10 years after surgery to verify that the patient is truly free of recurrence.

In conclusion, we suggest a MRI protocol that includes, a pre-operative MRI, and a 4- to 6-, 12- and 24-month post-operative MRI scan for every patient. When no tumoral residue is seen on these postoperative MRIs, the probability of tumoral regrowth is very low in which case pituitary radiotherapy is useless. MRI should be repeated 3, 5 and 10 years after the surgery in order to verify the absence of late recurrence. In patients with post-operative tumoral residue, we suggest that MRI must be repeated yearly. Because of its potential complications, radiotherapy should be considered only when the tumoral regrowth is proven except where the adenomatous residue is voluminous and close to the optic nerves/chiasma.

\section{References}

1 Gittoes NJL, Bates AS, Tse W, Bullivant B, Sheppard MC, Clayton $\mathrm{RN}$ et al. Radiotherapy for non-functioning pituitary tumours. Clinical Endocrinology 199848 331-337.

2 Turner HE, Stratton MI, Byrne JV, Adams CBT \& Wass JAH. Audit of selected patients with non-functioning pituitary adenomas treated without irradiation - a follow-up study. Clinical Endocrinology 199951 281-284.

3 Grigsby PW, Simpson JR \& Fineberg B. Late regrowth of pituitary adenomas after irradiation and/or surgery. Cancer 198963 1308-1312.

4 Brada M, Rajan B, Traish D, Ashley S, Holmes-Sellors PJ, Nussey S et al. The long term efficacy of conservative surgery and radiotherapy in the control of pituitary adenomas. Clinical Endocrinology 199338 571-578.

5 Tsang RW, Brierley JD, Panzarella T, Gospodarowicz MK, Sutcliffe SB \& Simpson WJ. Radiation therapy for pituitary adenoma; treatment outcome and prognostic factors. International Journal of Radiation Oncology Biology and Physics 199430 557-565.

6 Zierhut D, Flentje M, Adolph J, Erdman J, Raue F \& Wannmacher M. External radiotherapy of pituitary adenomas. International Journal of Radiation Oncology Biology and Physics 199533 $307-314$.

7 Fluckinger JC, Nelson PB, Martinez AJ, Deutsch M \& Taylor F. Radiotherapy of nonfunctional adenomas of the pituitary gland. Cancer $1996632409-2414$

8 Breen P, Flickinger JC, Kondziolka D \& Martinez AJ. Radiotherapy for nonfunctional pituitary adenomas: analysis of long term tumor control. Journal of Neurosurgery $1998 \mathbf{8 9} 933-938$.

9 Woollons AC, Hunn MK, Rajapakse YR, Toomath R, Hamilton DA, Conaglen J et al. Non-functioning pituitary adenomas: indications for postoperative radiotherapy. Clinical Endocrinology $200053713-717$.

10 McCord MW, Buatti JM, Fennell EM, Mendenhall WM, Marcus RB, Rhoton AL et al. Radiotherapy for pituitary adenomas: long-term outcome and sequelae. International Journal of Radiation, Oncology, Biology and Physics 199739 437-444. 
11 Rush S \& Cooper PR. Symptom resolution, tumor control, and side effects following post-operative radiotherapy for pituitary macroadenomas. International Journal of Radiation, Oncology, Biology and Physics 199737 1031-1034.

12 Al-Mejty O, Kersch JE, Routh A \& Smith RR. The long-term side effects of radiation therapy for benign brain tumors in adults. Journal of Neurosurgery $1990 \mathbf{7 3} 502-512$.

13 Brada M, Ford D, Ashley S, Bliss JM, Crowleys S, Masson M et al Risk of second brain tumour after conservative surgery and radiotherapy for pituitary adenomas. British Medical Journal 1992304 $1343-1346$

14 Tsang RW, Laperriere NJ, Simpson WJ, Brierley J, Panzarella T \& Smyth HS. Glioma arising after radiation therapy for pituitary adenoma. A report of four patients and estimation of risk. Cancer $1994722227-2233$.

15 Bradley KM, Adams CB, Potter CP, Wheeler DW, Anslow PJ \& Burke CW. An audit of selected patients with non-functioning pituitary adenoma treated by transsphenoidal surgery without irradiation. Clinical Endocrinology 1994 41 655-659.
16 Cottier J-P, Destrieux C, Brunereau L, Bertrand P, Moreau L, Jan M et al. Cavernous sinus invasion by pituitary adenoma. MR imaging. Radiology 2000215 463-469.

17 Kaplan EL \& Meier P. Non-parametric estimation for incomplete observations. Journal of the American Statistician Association $195853457-481$.

18 Steiner E, Math G, Knosp E, Mostbeck G, Kramer J \& Herold C. MR appearance of the pituitary gland before and after resection of pituitary adenomas. Clinical radiology 199449 524-530.

19 Rodriguez O, Mateos B, De la Pedraja R, Villoria R, Hernando JI, Pastor A et al. Postoperative follow-up of pituitary adenomas after transsphenoidal resection MRI and clinical correlation. Neuroradiology 199638 747-754.

Received 30 August 2001

Accepted 1 October 2001 